\title{
Multiband USB Antenna for Connecting Sensor Network and Internet
}

\author{
Wen-Shan Chen, Chien-Min Cheng, ${ }^{*}$ Yu-Liang Wang, and Guan-Quan Lin \\ Department of Electronic Engineering, Southern Taiwan University of Science and Technology, \\ No. 1, Nan-Tai Street, Yungkang Dist., Tainan City 710, Taiwan
}

(Received September 30, 2016; accepted January 6, 2017)

Keywords: coupled-fed antenna, monopole antenna, multiband USB antenna

In this paper, an antenna design with a multiband and a small size for connecting a sensor network and the Internet has been proposed. The antenna has an antenna portion of $20 \times 12 \mathrm{~mm}^{2}$ and a ground plane of $40 \times 12 \mathrm{~mm}^{2}$ which is printed on a flame retardant 4 (FR4) substrate $1.6 \mathrm{~mm}$ thick and with a relative dielectric constant of 4.4. A 50-ohm mini-coaxial cable is adopted to feed the RF input. The antenna portion consists of two meandered arms, and another small L-shaped arm with a chip inductor of $3.3 \mathrm{nH}$ is connected to the feed point of the design. A monopole with two meandered arms connected by a via hole is extended from the ground plane, which produces the modes of a low-frequency band. In addtion, the modes of high-frequency bands are also produced by these two meandered arms and the ground plane. The simulated and measured results of the proposed antenna cover the bands of LTE700/LTE2300/LTE2500/GSM1800/GSM1900/ UMTS. This USB antenna design with multibands and a small size is suitable for connecting a sensor network and the Internet.

\section{Introduction}

The number of products for wireless communication has dramatically increased worldwide. It is expected that wireless products will have more and more applications and seamless connectivity to the Internet. The data collected from a sensor network should be transmitted to the Internet for data processing and analysis. Good, high-speed connectivity to the Internet is also expected for most applications. Therefore, enhanced spectrum efficiency and transmission rate are needed in wireless technology and development. How to include more frequency bands in the system is an important issue for researchers.

Recently, the next-generation $4 \mathrm{G}$ mobile wireless broadband communication system has been long term evolution (LTE), which was developed by the 3rd generation partnership project (3GPP). Chen et al. ${ }^{(1)}$ used two curve strips to design LTE 700 single band. Li et al. ${ }^{(2)}$ adopted a simple structure to design an antenna for GSM900/DCS1800/PCS1900/UMTS2100/LTE2300/2500 bands. Sun and Ban and their coworkers ${ }^{(3,4)}$ designed an eight-band (LTE/GSM/UMTS) antenna with two surface mount technology (SMT) devices having dimensions of $70 \times 20 \times 0.8 \mathrm{~mm}^{3}$, and the antenna portion was only $19 \times 20 \mathrm{~mm}^{2}$. It was found that for a dongle antenna, covering all the bands of LTE/GSM/UMTS is not an easy task. Therefore, in this paper, a six-band (LTE700/DCS1800/ PCS1900/UMTS2100/LTE2300/2500) miniature dongle antenna is proposed and investigated.

*Corresponding author: e-mail: ccmin523@gmail.com http://dx.doi.org/10.18494/SAM.2017.1530 


\section{Antenna Design and Application Bands}

The proposed antenna is fabricated on a flame retardant 4 (FR4) substrate $1.6 \mathrm{~mm}$ thick with a dielectric constant of 4.4. Figure 1 shows the geometry of the proposed antenna; the overall area of the design is $60 \times 12 \mathrm{~mm}^{2}$. In addition, the size of the antenna portion is only $20 \times 12 \mathrm{~mm}^{2}$. The detailed dimensions of the design are listed in Table 1. A 50-Ohm coaxial cable as RF input is connected to a small L-shaped arm to couple the signal to this antenna with two meandered arms. A chip inductor of $3.3 \mathrm{nH}$ is adopted to improve the impedance of all operating bands. The two meandered arms are connected by a via hole. The meandered arm with the longest length on the front side of the substrate produces the mode of $0.735 \mathrm{GHz}$; the meandered arm extended from the front side and connected by a via hole with the second longest length produces the mode of 0.9 GHz. The two meandered arms extended from the ground plane produce the two modes for the low-frequency band. The higher modes of these two meandered arms and the mode of the ground plane form the mode of the high-frequency band.

Figure 2 shows the simulated and measured S11 of the proposed antenna. The simulated results are obtained by high frequency structure simulation (HFSS). The simulated and measured results are in good agreement to verify the reliability of the HFSS software and proposed antenna. The measured results with the bands of $0.696-0.788$ and $1.69-2.83 \mathrm{GHz}$ can cover the six bands of LTE700/LTE2300/LTE2500/GSM1800/GSM1900/UMTS. The design with multibands and a small size can be used for USB dongle applications.
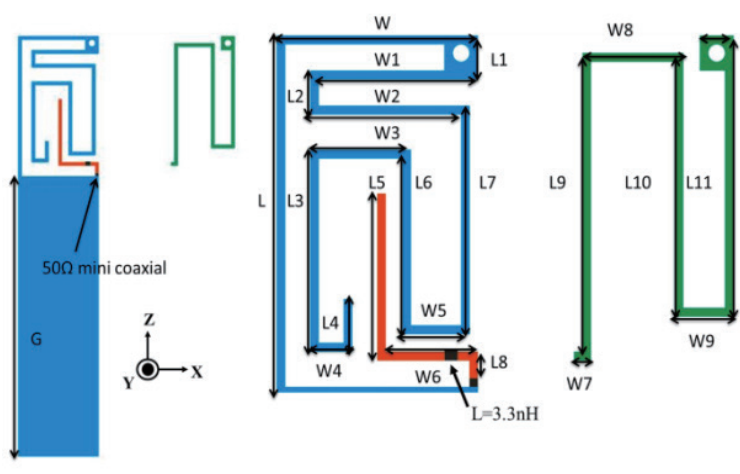

Fig. 1. (Color online) Geometry of the proposed antenna.

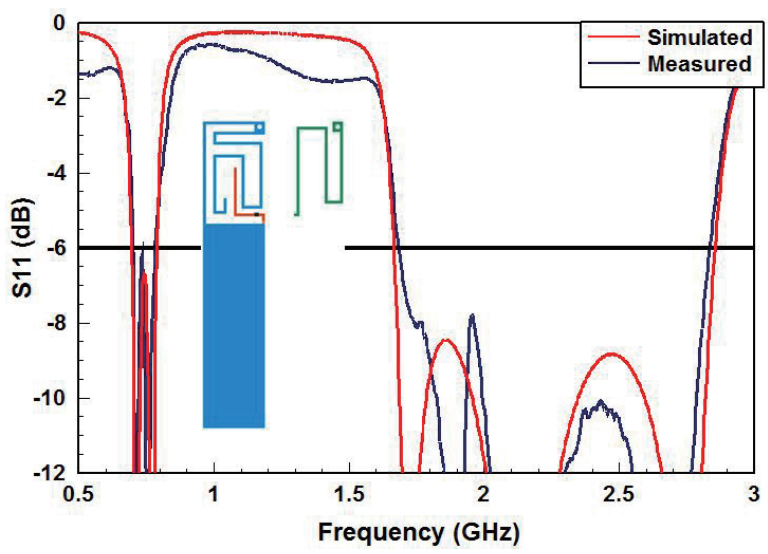

Fig. 2. (Color online) Simulated and measured S11 of the proposed antenna.

Table 1

Detailed dimensions of the proposed antenna.

\begin{tabular}{cccccccc}
\hline Parameters & Unit $(\mathrm{mm})$ & Parameters & Unit $(\mathrm{mm})$ & Parameters & Unit $(\mathrm{mm})$ & Parameters & Unit $(\mathrm{mm})$ \\
\hline L & 20 & L7 & 13 & W & 12 & W7 & 1 \\
L1 & 2.5 & L8 & 1.5 & W1 & 9.5 & W8 & 6 \\
L2 & 2.5 & L9 & 17 & W2 & 9 & W9 & 3.5 \\
L3 & 11.5 & L10 & 14.5 & W3 & 6 & W10 & 2 \\
L4 & 3 & L11 & 16 & W4 & 2.5 & & \\
L5 & 9.5 & G & 40 & W5 & 4 & \\
L6 & 10 & & & W6 & 5.5 & & \\
\hline
\end{tabular}




\section{Results and Discussion}

The antenna parametric studies are discussed in this section. The simulated S11 and impedance of the proposed antenna with different L5 lengths are shown in Fig. 3. It can be clearly observed that, as the L5 length decreases to $5.5 \mathrm{~mm}$, the low-frequency mode of the antenna shifts gradually to higher frequency.

Figure 4 shows the simulated S11 parameters and the impedance of the proposed antenna with different $\mathrm{AB}$ lengths. It can be observed from these two figures that, as the $\mathrm{AB}$ length decreases from 23 to $3 \mathrm{~mm}$, the second low-frequency mode of the antenna shifts to a higher frequency and the first high-frequency mode of the antenna shifts to a higher frequency.

Figure 5 shows the simulated S11 parameters and impedance of an antenna with different CD lengths. As the CD length decreases to $12 \mathrm{~mm}$, the second low-frequency mode moves to a higher frequency. Moreover, from the impedance variation of the design with different CD lengths, it can be found that the first low-frequency mode of the antenna shifts slightly to a higher frequency and the second low-frequency mode of the antenna shifts to a higher frequency as the CD length increases.

Figure 6 shows the simulated S11 parameters and impedance of an antenna with different G lengths (the length of the ground plane). It can be observed that as the $\mathrm{G}$ length increases from 40
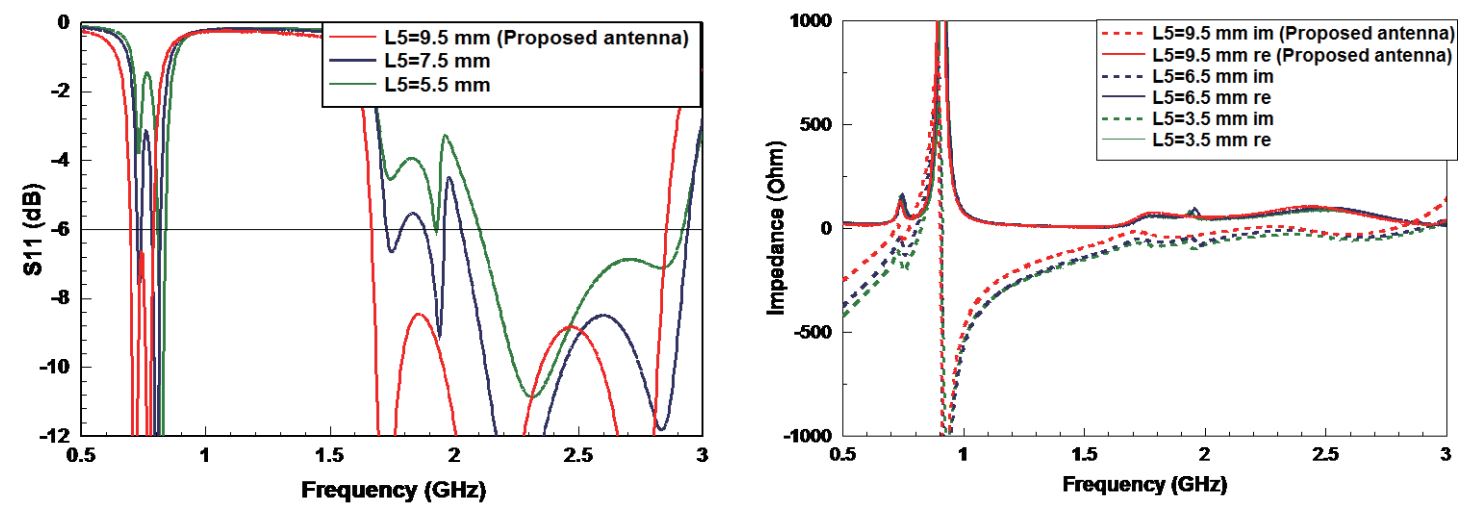

Fig. 3. (Color online) Simulated S11 and impedance of an antenna with different lengths of L5.
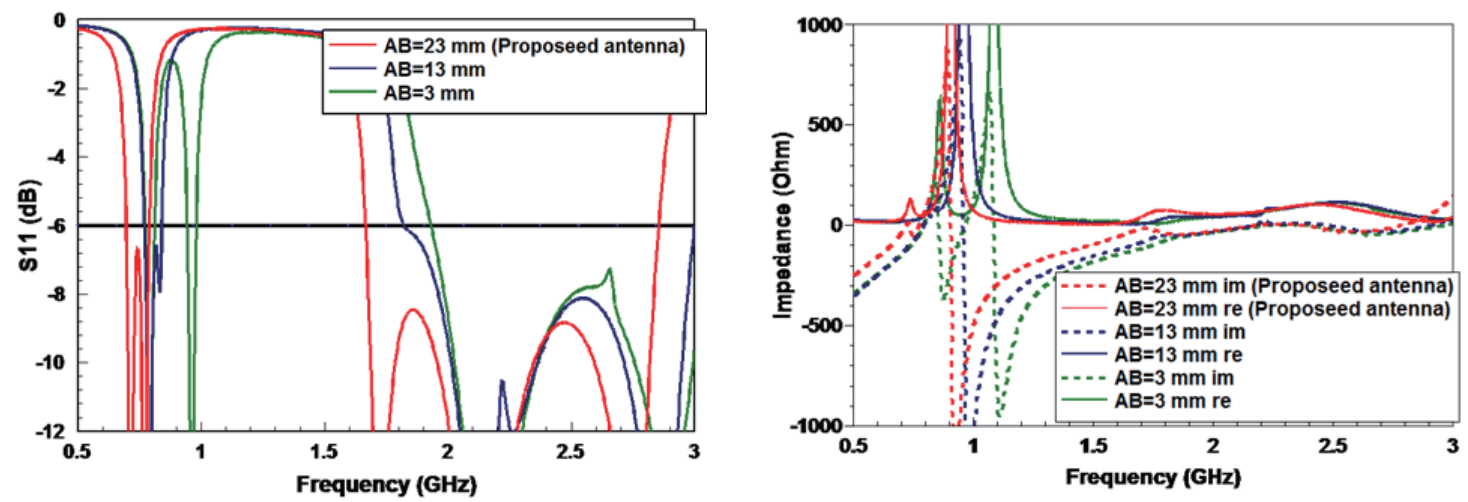

Fig. 4. (Color online) Simulated S11 and impedance of an antenna with different lengths of AB. 

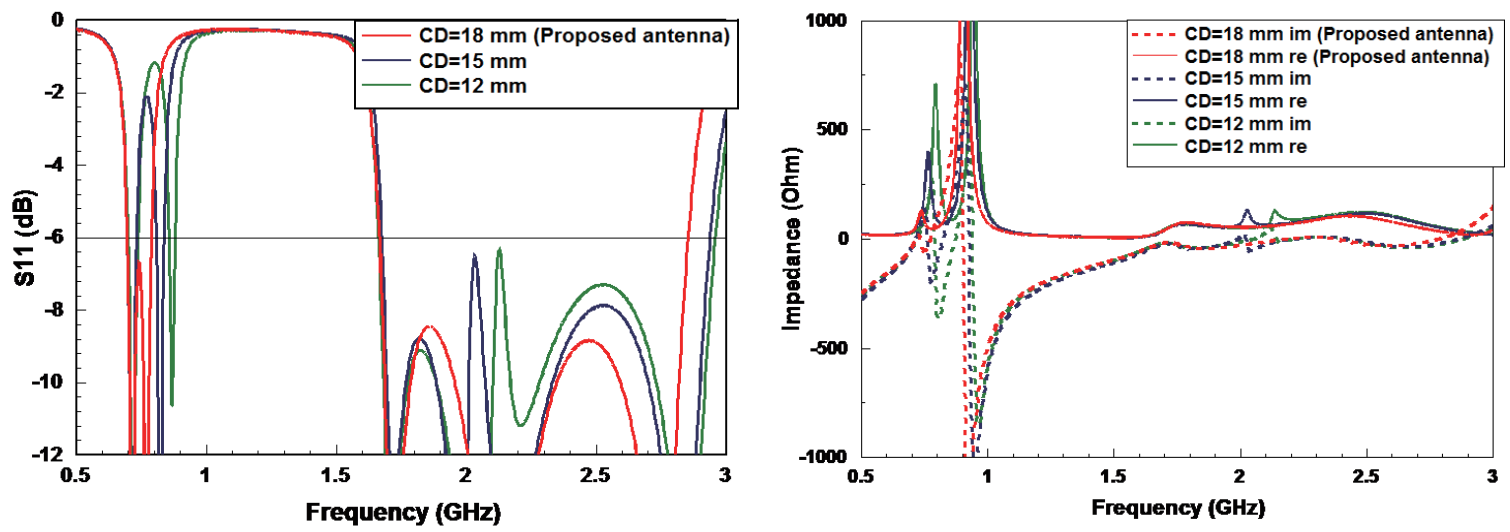

Fig. 5. (Color online) Simulated S11 and impedance of an antenna with different lengths of CD.
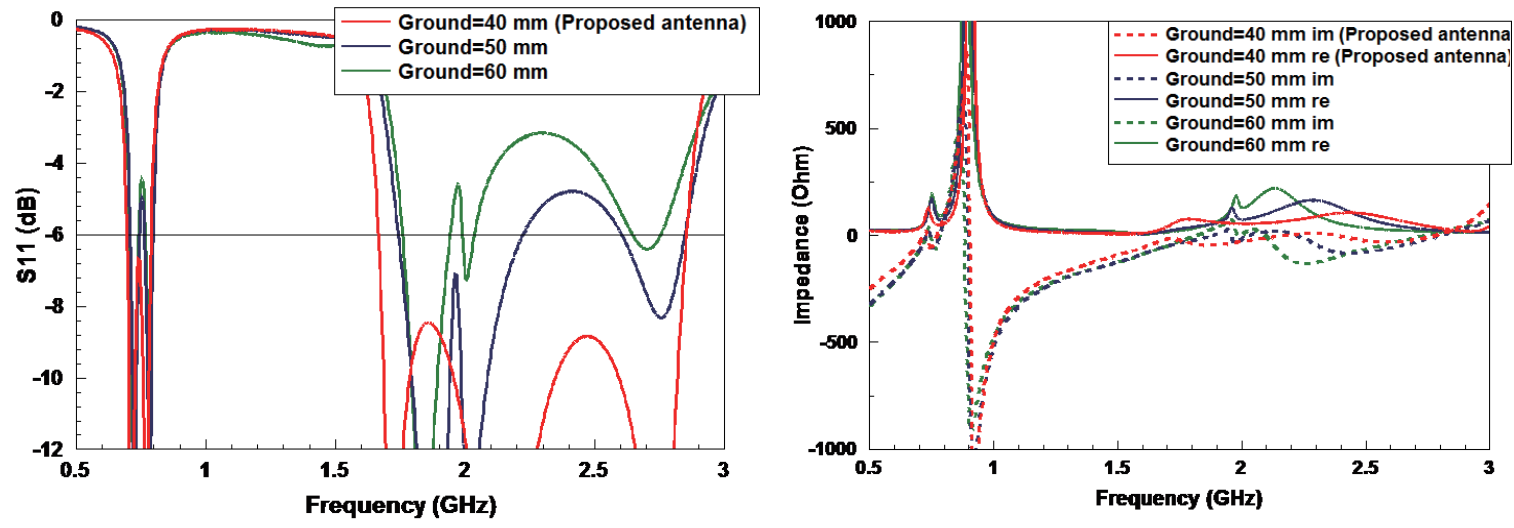

Fig. 6. (Color online) Simulated S11 and impedance of an antenna with different lengths of G.
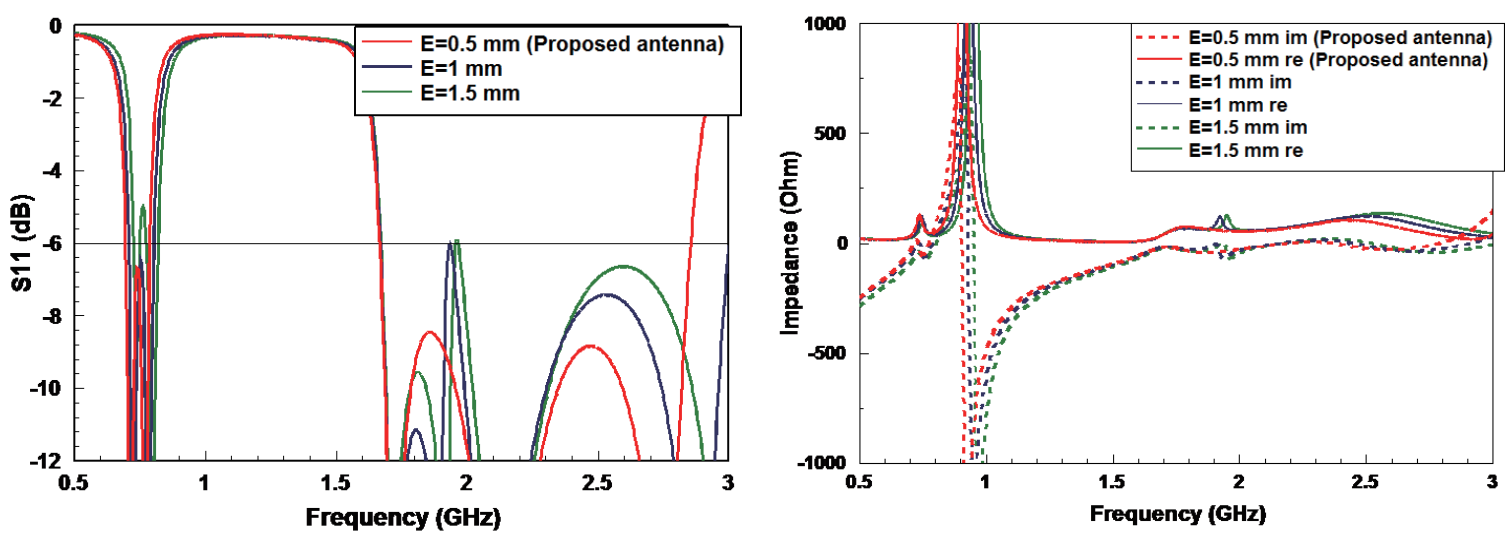

Fig. 7. (Color online) Simulated S11 and impedance of an antenna with different lengths of E.

to $60 \mathrm{~mm}$, the band of $2.0-2.5 \mathrm{GHz}$ is strongly affected. Moreover, from the impedance variation plot shown, considerable variations can also be seen between 2.0 and $2.5 \mathrm{GHz}$.

Figure 7 shows the simulated S11 parameters and impedance of an antenna with different $\mathrm{E}$ widths. As the E length increases from 0.5 to $1.5 \mathrm{~mm}$, the mode $(2.5 \mathrm{GHz})$ of the design shifts to a 
higher frequency. Moreover, for the impedance of the design with different $\mathrm{E}$ widths, the mode (2.5 $\mathrm{GHz}$ ) of the design shifting to a higher frequency can also be observed.

The radiation patterns, antenna gain, and efficiency of the antenna are also measured and discussed. Figures 8(a)-8(e) depict the measured 2-D radiation patterns of $0.715,0.76,1.75$,

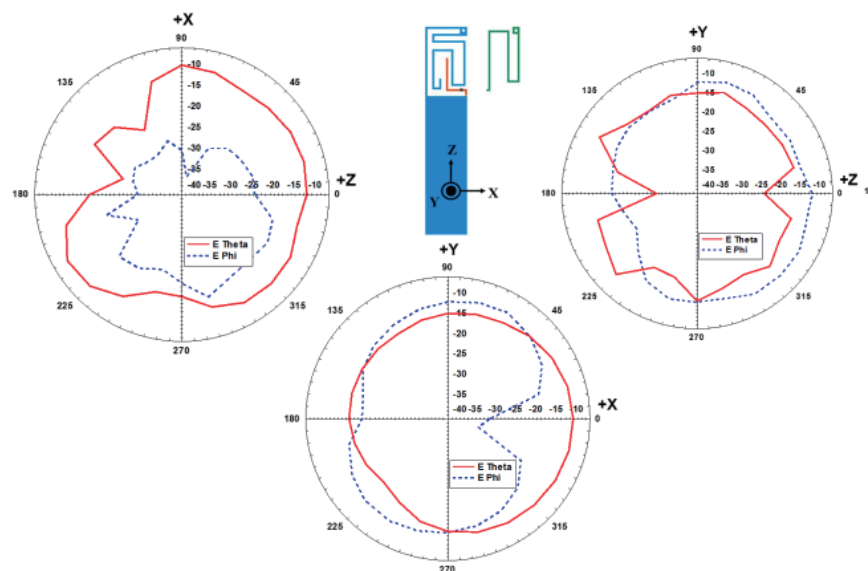

(a)

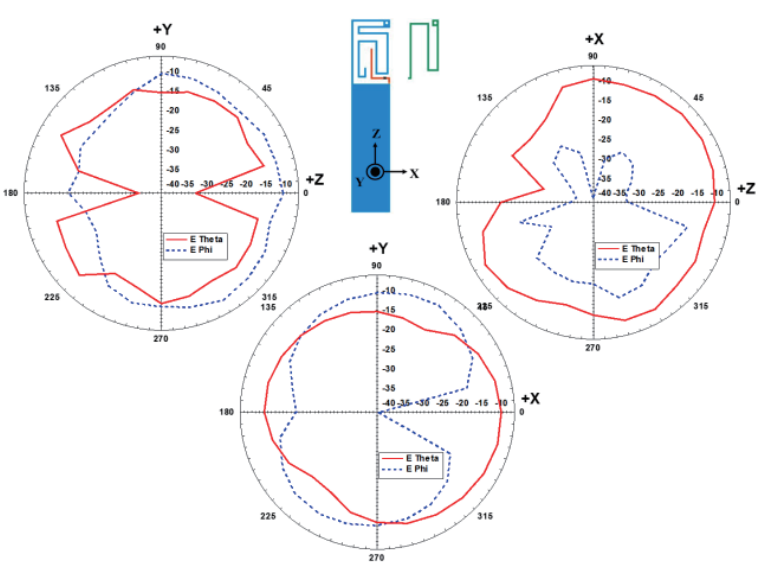

(b)

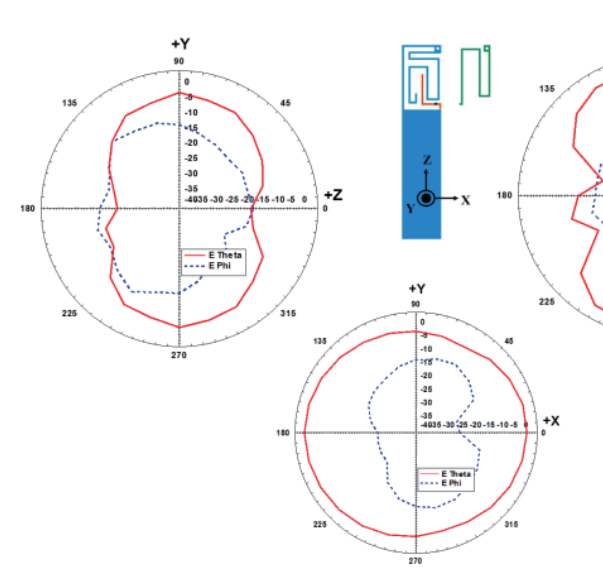

(c)

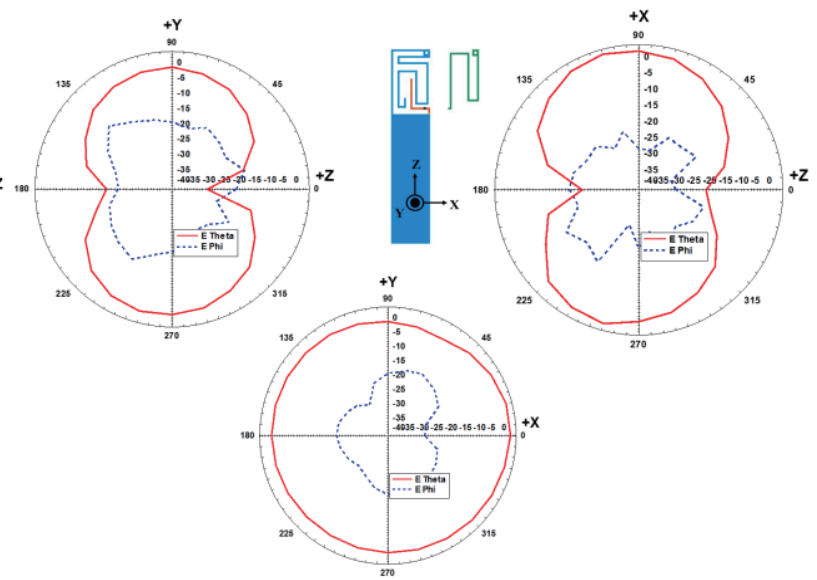

(d)

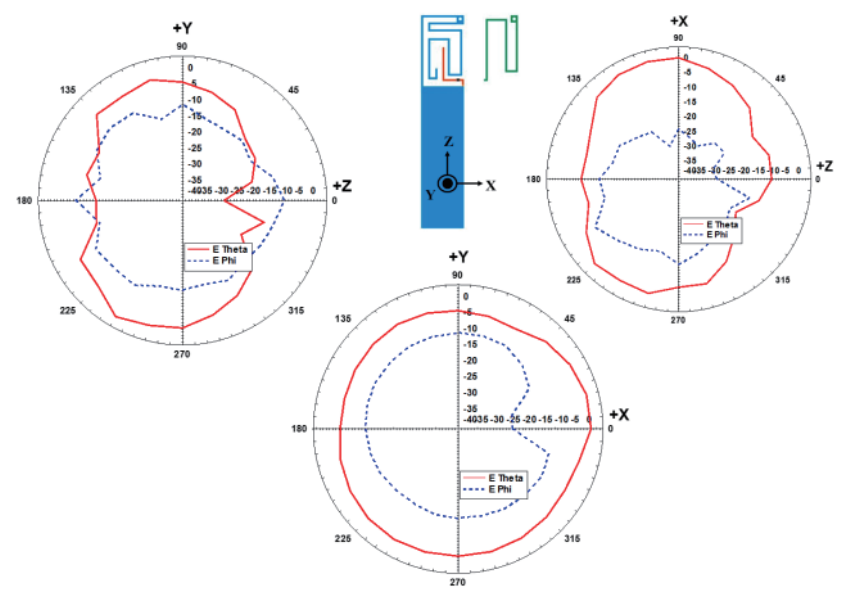

(e)

Fig. 8. (Color online) Measured 2-D radiation patterns of $Y-Z, X-Z$, and $X-Y$ planes: (a) 0.715, (b) 0.76, (c) 1.75, (d) 2.137 , and (e) $2.7 \mathrm{GHz}$. 


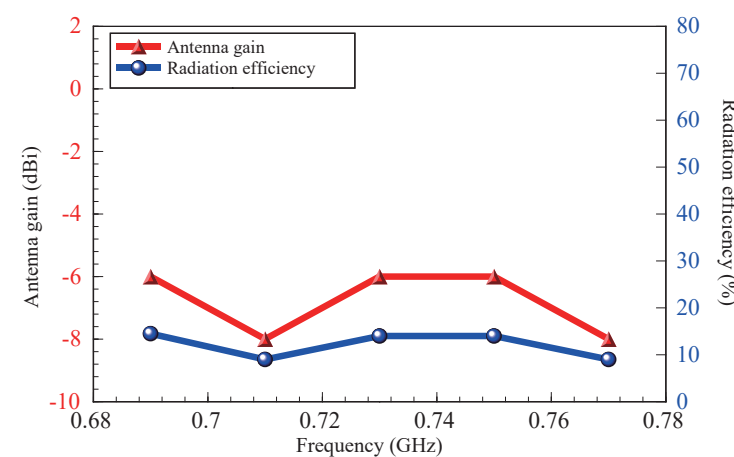

(a)

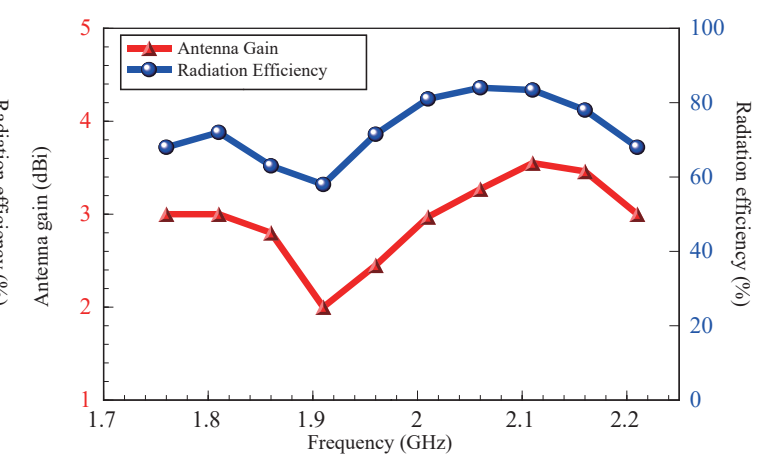

(b)

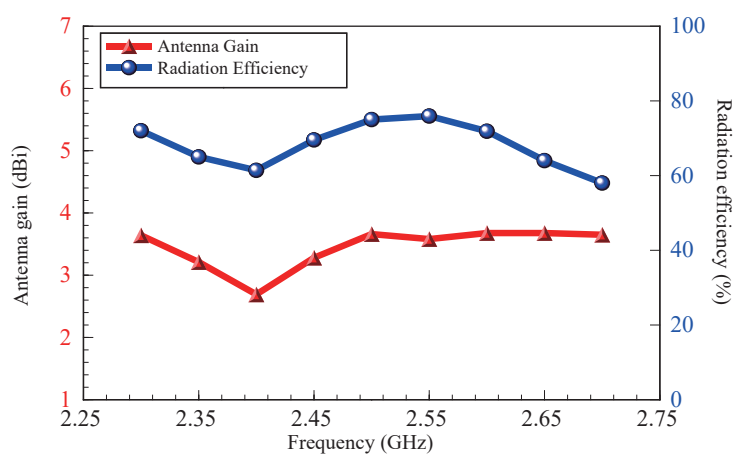

(c)

Fig. 9. (Color online) Measured antenna gains and radiation efficiencies: (a) 0.69-0.77, (b) 1.75-2.2, and (c) 2.3-2.7 $\mathrm{GHz}$.

2.137, and $2.7 \mathrm{GHz}$, respectively. It can be observed that for all these frequencies, the $X-Y$ plane radiations reveal nearly omnidirectional characteristics for both low as well as high frequencies.

Figures 9(a)-9(c) show the measured antenna gain and radiation efficiency of the antenna for $0.69-0.77,1.75-2.2$, and $2.3-2.7 \mathrm{GHz}$ bands, respectively. The measured antenna gains vary between 0.024 and $0.138 \mathrm{dBi}$ and the radiation efficiency varies between 4 and $20 \%$ for the lowfrequency band. On the other hand, the measured antenna gains vary between 1.57 and $2.08 \mathrm{dBi}$ and the radiation efficiency varies between 60 and $92 \%$ for the high-frequency band. From the above results, the impedance and radiation performances of the proposed antenna are good for practical applications.

\section{Conclusions}

A dongle antenna with six bands of LTE/GSM/UMTS for connecting a sensor network and the Internet has been proposed and examined. The design using coupled-fed meander arms covers the design goal of LTE700/LTE2300/LTE2500/GSM1800/GSM1900/UMTS (0.698-0.787 $\mathrm{GHz} / 1.71-2.17 \mathrm{GHz} / 2.3-2.4 \mathrm{GHz} / 2.5-2.69 \mathrm{GHz}$ ) bands. The performance of the proposed antenna was confirmed on the basis of simulated and measured results. The antenna design with a simple structure, small size, and good radiation performance is a good candidate for devices to connect to the Internet. 


\section{Acknowledgements}

This work was kindly supported by the Ministry of Science and Technology of the Republic of China (MOST 105-2221-E-218-034 and 105-ET-E-218-001-ET).

\section{References}

1 W. S. Chen and C. Y. Huang: 2015 IEEE Int. Symp. Antennas Propag. \& USNC/URSI National Radio Sci. Meeting, p. 1146.

2 P. Li, Z. Nie, X. Zong, J. Ouyang, and Y. Ban: 2011 Cross Strait Quad-Regional Radio Sci. Wirel. Technol. Conf. (CSQRWC) p. 926.

3 S. C. Sun, Y. L. Ban, C. L. Liu, and J. L. W. Li: 2012 Int. Symp. Antennas, Propag. \& EM Theory (ISAPE2012) p. 86.

4 Y. L. Ban, J. H. Chen, S. C. Sun, and J. L. W. Li: IEEE Antennas Wirel. Propag. Lett. 11 (2012) 767.

\section{About the Authors}

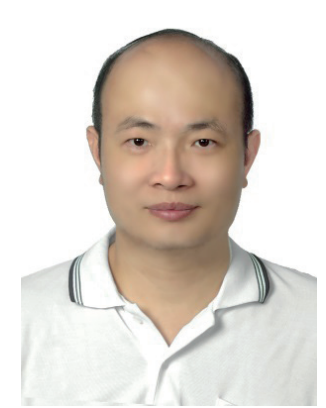

Wen-Shan Chen received his B.S. degree from National Taiwan University of Science and Technology in 1990 and his Ph.D. degree from National Sun Yat-Sen University, Taiwan, in 2001. He is currently a Professor of Southern Taiwan University of Science and Technology. He is an IEEE Senior Member with AP, MTT, and EMC Societies. He served as the General Secretary in 2007-2008, Vice Chair in 2009-1010, and Chair in 2011-2012, all with the IEEE AP-S Tainan Chapter. He is also a consultant of IEEE AP-S Tainan Chapter. $\mathrm{He}$ is a member of the Institute of Antenna Engineers of Taiwan and was elected to be an AdCom member in 2008-2015. He is a member of the Chinese Microwave Association and was elected to be an AdCom member in 20152016. He is a member of the TIEEE, Chinese Institute of Electrical Engineering, and IEICE. His research interests include antenna design, $\mathrm{RF}$, and microwave circuits
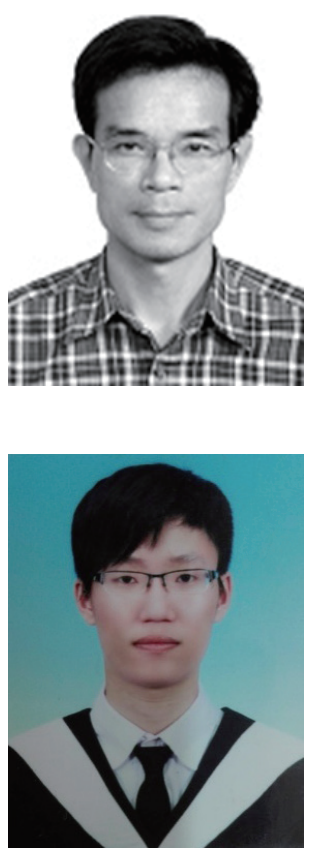

Chien-Min Cheng received his B.S., M.S., and Ph.D. degrees from the National Taiwan Ocean University, National Cheng Kung University, and National Sun Yat-Sen University, Taiwan, in 1984, 1990, and 2008, respectively. From 2009 to 2012, he was an associate professor of Southern Taiwan University of Science and Technology, Taiwan. Since 2013, he has been a professor at the same university. His research interests are in electronic ceramics, thin films, microwave device design, and piezoelectric ceramics.

Yu-Liang Wang was born in Kaohsiung, Taiwan, in 1993. He received his B. S. degree from the Department of Electric Engineering, Southern Taiwan University of Science and Technology, Tainan, Taiwan, in 2015. His research interests are in USB dongle antennas, couple fed monopole antennas, and multiband antennas. 


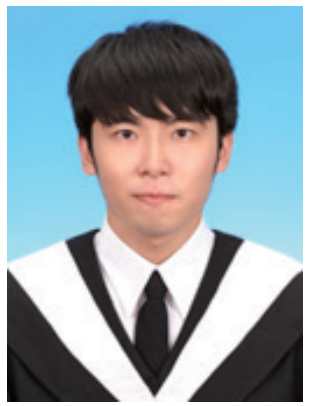

Guan-Quan Lin was born in Taichung, Taiwan, in 1992. He received his B.S. degree from the Department of Electric Engineering, Southern Taiwan University of Science and Technology, Tainan, Taiwan, in 2015. He is currently pursuing his M.S. degree at Southern Taiwan University of Science and Technology, Tainan. His research interests include USB dongle antennas and wearable device antennas. He is currently focusing on research on smart watch antennas on watch heads. 